\title{
Impacto de dos arrecifes artificiales en la pesca artesanal diurna del Golfo de Morrosquillo, Caribe de Colombia
}

\author{
Oscar Delgadillo-Garzón ${ }^{1} \&$ Camilo B. García ${ }^{2}$ \\ 1. MoAm Monitoreos Ambientales. Calle 127 \#53 -68, Apto 502, Bogotá, Colombia; oskadelgadillo@gmail.com \\ 2. Universidad Nacional de Colombia, Departamento de Biología, Bogotá, Colombia; cbgarciar@unal.edu.co
}

Recibido 20-XI-2009. Corregido 10-IV-2009. Aceptado 12-V-2009.

\begin{abstract}
Impact of two artificial reefs on diurnal artisanal fishing at gulf of Morrosquillo, Colombian Caribbean. Fishing activity in two artificial reefs (ARs) was monitored between August 2001 and August 2002 in the gulf of Morrosquillo, to investigate their possible impact on the fishery dynamics. We determined catch per unit effort (CPUE) and catch composition, and found 19 species in Punta de Piedra and 36 in Tolú (16 species common to both). In Punta de Piedra, CPUE was $0.40 \mathrm{~kg} /$ hour/fisherman and estimated annual catch was 1830 $\mathrm{kg}$ (Tolú values: $0.42 \mathrm{~kg} /$ hour/fisherman and $4820 \mathrm{~kg}$ ). No significant differences in CPUE were found between ARs and no seasonal pattern was evident. The differences in total composition and extracted biomass between ARs were mainly a consequence of greater effort exerted in Tolú, whereas the absence of trends and correlation with environmental factors of CPUE and species caught suggests that the ARs are near their carrying capacity. As expected, the ARs supported and improved CPUE and fishing success in comparison with reported values of natural habitats, traditional fishing grounds and some artificial habitats in the zone, owing to their greater volume, structural complexity and vertical profile. However, this increase could also be the result of biomass aggregation, not production. The potential negative impacts of ARs on the environment require experimental research. The deployment of non-extractive ARs and the controlled usage for fishery purposes could be a useful tool for resource enhancement and management in the area. Rev. Biol. Trop. 57 (4): 993-1007. Epub 2009 December 01 .
\end{abstract}

Key words: artificial reefs (ARs), catch per unit effort (CPUE), fishery dynamics, gulf of Morrosquillo, Colombia, Caribbean Sea.

La disminución en los recursos pesqueros es evidente y es el resultado de la sobrepesca, el manejo insostenible y los cambios ecológicos imprevistos, así como de la degradación y los disturbios ambientales (Botsford et al. 1997, FAO 1997, Pauly et al. 1998, Essington et al. 2006). El Golfo de Morrosquillo, como otros sectores costeros del mundo, ha estado expuesto a una constante presión ambiental debido a las actividades portuarias, contaminación, turismo y pesca extensiva, lo que ha causado una reducción en las capturas y modificación de los ecosistemas, amenazando la sostenibilidad de los recursos a largo plazo (Ramírez et al. 1994, SEA 2000, CIOH 2001).
Una opción para mejorar la producción pesquera ha sido el uso de los arrecifes artificiales (AAs). Sin embargo, la pregunta de si las estructuras artificiales incrementan, reducen o simplemente redistribuyen los recursos pesqueros, es todavía ampliamente debatida (Pickering \& Whitmarsh 1997, Seaman \& Jensen 2000). De igual forma, pese a que la construcción de AAs para actividades pesqueras y buceo recreativo ha recibido un gran soporte público, la creación de AAs no extractivos, i.e., para la conservación de los recursos, educación o investigación, es esencialmente inexistente (Pitcher \& Seaman 2000, Bohnsack 2005). 
En el Caribe de Colombia se han instalado algunos AAs para mejorar las actividades pesqueras, pero su éxito e impacto es poco conocido debido al escaso esfuerzo de monitoreo. Ejemplos son los trabajos de García \& Navarro (1998), con dispositivos agregadores de peces (DAPs) en Isla Aguja (Santa Marta), Castro \& Stephens (2000), en la Isla de San Andrés y la instalación de AAs que se realizó en La Guajira por la ONG Terrazul desde el año 2003 (Terrazul 2006). En el Golfo de Morrosquillo, Herazo \& Torres (1998), evaluaron la efectividad de AAs de llantas, con una captura principalmente compuesta por especies de importancia comercial, mientras que en DAPs dispuestos en aguas costeras del mismo sector, Gámez \& Sáenz-Betancourt (2001), registraron individuos juveniles de especies comerciales. Estas investigaciones, si bien aluden al tema pesquero, fueron realizadas en períodos cortos de tiempo, por lo cual no se determinaron los impactos de las estructuras sobre la pesquería en su área de influencia.

Delgadillo et al. (2004) y Delgadillo (2005) evaluaron la dinámica del ensamblaje de peces e invertebrados asociados a dos nuevos AAs tipo fortaleza por medio de censos visuales.
Esta investigación se enfoca particularmente en la actividad pesquera artesanal diurna, ejercida en el curso de un año en dichos AAs, con el objetivo de determinar las posibles diferencias en la captura por unidad de esfuerzo (CPUE) y en el tamaño promedio de las especies comerciales capturadas: 1) entre los dos AAs (diferencias en localización y volumen), 2) entre los AAs con respecto a otros hábitats artificiales y naturales del sector, 3) con los datos pesqueros antes de la instalación de los AAs, y 4) con el cambio de estación seca a lluviosa y otros factores ambientales, como insumos en el proceso de conocer y entender el funcionamiento de los AAs en el golfo.

\section{MATERIALES Y MÉTODOS}

Área de estudio: El Golfo de Morrosquillo, localizado a los $9^{\circ} 22^{\prime}-9^{\circ} 45^{\prime} \mathrm{N}$ y $75^{\circ} 33^{\prime}$ $75^{\circ} 55^{\prime} \mathrm{W}$ ocupa un área de aproximadamente $1000 \mathrm{~km}^{2}$ (Fig. 1A), con profundidades entre $0 \mathrm{~m}$ y $55 \mathrm{~m}$, y una plataforma de $70 \mathrm{~km}$ de máxima extensión en la parte más ancha $(\mathrm{CIOH}$ 2001). El golfo está rodeado por manglares y estuarios; en sus límites hay arrecifes de coral,
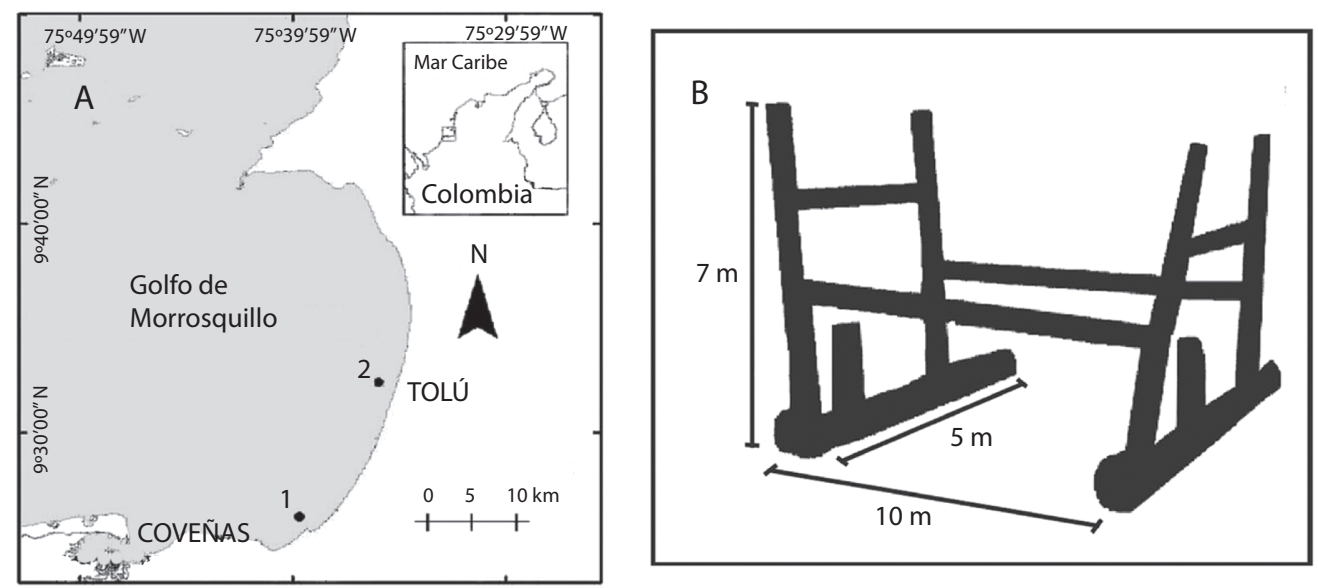

Fig. 1. A: Golfo de Morrosquillo con la ubicación esquemática de los AAs (1: Punta de Piedra; 2: Tolú). B: Esquema del diseño de los AAs con las respectivas dimensiones.

Fig 1. A: Gulf of Morrosquillo with the location of the ARs sites (1: Punta de Piedra; 2: Tolú). B: Design scheme of the AR with the respective dimensions. 
pastos marinos y en el extremo suroeste se encuentra la bahía de Cispatá, antiguo delta del río Sinú, con aproximadamente 4 622ha de manglar y un sistema estuarino de gran influencia en el medio marino, en cuanto a los aportes fluviales y sus efectos en la salinidad superficial en la época invernal (Patiño \& Flórez 1993, Kochane 2000, Sánchez-Páez et al. 2005).

El 80\% del fondo marino está cubierto por material calcáreo cuya proporción se reduce hacia la costa por efecto del río Sinú y otros ríos que fluyen en el golfo. Se distingue una época seca de diciembre hasta abril, y una húmeda de mayo a noviembre, con una reducción de precipitaciones entre junio y julio (verano). La temperatura del agua superficial oscila entre $26^{\circ} \mathrm{C}$ y $32^{\circ} \mathrm{C}$, y la salinidad entre 24 y 35 (Molina et al. 1994, Delgadillo et al. 2004).

Detalles de los arrecifes artificiales: Los AAs fueron construidos con tubería de oleoducto en acero y mangueras submarinas de caucho elástico, formando el esqueleto de un cubo rectangular (10m x 5m x 7m) (Fig. 1B). Dentro de la estructura se colocaron piezas del mismo material sin una disposición regular, incrementando la complejidad estructural del hábitat. Un AA se instaló en el sector de Punta de Piedra y el otro en Tolú. Punta de Piedra tuvo un volumen de $200 \mathrm{~m}^{3}$, fue instalado a $1.6 \mathrm{mi}-\mathrm{n}$ de la costa y $20 \mathrm{~m}$ de profundidad, a los $9^{\circ} 27^{\prime} 30^{\prime \prime} \mathrm{N}$ y $75^{\circ} 40^{\prime} 42^{\prime}$ ' W. Tolú presentó $138 \mathrm{~m}^{3}$ de volumen, fue dispuesto a $0.97 \mathrm{mi}-\mathrm{n}$ de la costa a una profundidad de $16 \mathrm{~m}$, en las coordenadas $9^{\circ} 31^{\prime} 30^{\prime \prime} \mathrm{N}$ y $75^{\circ} 37^{\prime} 24$ ' W (Fig. 1A). Cada estructura fue marcada con una boya blanca asida a la estructura por una cadena.

Métodos de muestreo y análisis de la información: Desde agosto del 2001 hasta agosto del 2002 se realizó el seguimiento de la actividad pesquera tanto en Tolú como en Punta de Piedra, excluyendo febrero y marzo debido a dificultades logísticas. Se debe anotar que en noviembre, diciembre, abril y julio, Punta de Piedra no fue visitado por los pescadores en razón al aumento de los vientos (noviembre/diciembre) y la pesca concurrente con trasmallos, redes de arrastre y buzos arponeros. Los datos de captura y esfuerzo (CPUE) fueron muestreados entre tres y siete días del mes escogidos aleatoriamente para cada AA, algunos días simultáneamente y otros en cada AA por separado. En el puerto de desembarco (parte sur de Tolú y playa entre Punta de Piedra y Coveñas) se recolectó información sobre el número de canoas, pescadores por canoa, hora de salida y llegada, así como la composición, abundancia, peso y longitud estándar (LE) de la captura, según la metodología descrita en Bortone et al. (2000). Este estudio se restringió a pescadores que utilizaban canoa de madera, remo, vela, línea de mano, carnada viva, y que pescaban únicamente durante las horas del día.

Antes de iniciar la evaluación, una serie de entrevistas realizadas a los pescadores permitieron calcular los días efectivos de pesca a la semana (6), valor con el cual se estimó la biomasa total extraída en el año para cada AA. La captura diaria fue promediada por el número de pescadores-horas del día (desde el tiempo de salida al tiempo de llegada, para considerar una medida completa de esfuerzo pesquero) y este valor se utilizó para comparar la CPUE entre sitios y épocas climáticas.

Para los análisis, los datos de CPUE se transformaron a logaritmo, pero como no se ajustaron a una distribución normal, se realizó la prueba de Levene $(\mathrm{W}=0.31 ; \mathrm{p}>0.05)$, con la cual se rechazó la hipótesis de diferencias en varianzas. Seguidamente, se hizo el análisis de varianza (ANOVA) convencional el cual es legítimo, bajo el supuesto de que la falta de normalidad no necesariamente induce a rechazar una hipótesis nula cuando existe homogeneidad de varianzas (Underwood 1997). De esta forma, se hizo ANOVA de una sola vía (Zar 1996) para comparar los valores de CPUE entre Punta de Piedra y Tolú en la época de lluvia debido a la ausencia de desembarcos durante la época seca en Punta de Piedra. La misma prueba se utilizó para comparar la CPUE entre épocas climáticas solo para Tolú bajo los condicionamientos relatados arriba que impidieron un análisis de varianza de dos vías (AAs y 
épocas climáticas). De igual forma, los valores de CPUE (el promedio mensual) en los AAs fueron comparados con ANOVA de una vía con la CPUE mensual de anteriores estudios en el sector (unidades comparables o modificadas para asegurar la comparabilidad), provenientes del mismo tipo de pesca realizada durante el día en hábitats naturales, caladeros tradicionales de pesca y otros hábitats artificiales.

Los valores de longitud estándar (LE) de las especies abundantes y comerciales se compararon entre AAs para la época lluviosa, y entre épocas para el AA de Tolú, en ambos casos con la prueba de Kruskal-Wallis, debido a que los datos no cumplieron los supuestos de normalidad y homogeneidad de varianzas. Se realizó un análisis de clasificación (índice de Bray-Curtis, técnica de ligamento completo) de los meses del monitoreo, utilizando como atributo la CPUE mensual por especie. Se correlacionaron por rangos de Spearman los aspectos biológicos CPUE (promedio diario) y composición de la captura (número diario de especies) con los factores abióticos (temperatura y salinidad) (Ludwig \& Reynolds 1988, Clark \& Warwick 2001). Valores in situ de temperatura y salinidad fueron medidos con un conductímetro ATC ${ }^{\circledR}$ con precisión de $1 \%$, a un metro de profundidad del sector donde estaban instaladas las estructuras, entre las 10am y $12 \mathrm{pm}$ concurrente con cada día de muestreo. Todas las pruebas estadísticas se hicieron utilizando los programas Biodiversity Professional $2 ®$ y Statgraphics Plus 5.0®.

\section{RESULTADOS}

Durante el período de muestreo se registró un total de 39 especies, 19 en Punta de Piedra y 36 en Tolú, con 16 especies en común (Cuadro 1). Considerando los días efectivos de pesca en el seguimiento, la biomasa extraída anual aproximada en los AAs fue de $1830 \mathrm{~kg}$ en Punta de Piedra y $4820 \mathrm{~kg}$ en Tolú. Igualmente, el tiempo estimado de pesca sobre las estructuras fue de 5494 horas en Punta de Piedra y 11213 horas en Tolú, obtenido de un total de 64 y 333 faenas respectivamente, con una intensidad pesquera substancialmente mayor en Tolú. En el año, 34 unidades pesqueras o canoas visitaron Punta de Piedra y 26 Tolú, con promedios diarios de $2.68(\mathrm{DE}=2.09)$ y 3.58 $(\mathrm{DE}=2.33)$, respectivamente.

El promedio de CPUE durante el período de estudio en Punta de Piedra fue de $0.40 \mathrm{~kg} /$ hora/pescador ( $\mathrm{n}=24$ días monitoreados de pesca; $\mathrm{DE}=0.23$ ) con ámbito entre $0.10 \mathrm{~kg}$ / hora/pescador y $0.88 \mathrm{~kg} /$ hora/pescador. En Tolú, el promedio de CPUE fue de $0.42 \mathrm{~kg} /$ hora/pescador ( $\mathrm{n}=93$ días monitoreados de pesca; $\mathrm{DE}=0.25$ ) y osciló entre $0.03 \mathrm{~kg} / \mathrm{hora} /$ pescador y $1.28 \mathrm{~kg} /$ hora $/$ pescador (Fig. 2). El mes que presentó mayor CPUE en Punta de Piedra fue agosto de $2001(0.62 \mathrm{~kg} / \mathrm{hora} /$ pescador, $\mathrm{DE}=0.32$, promedio mensual) y en Tolú fue noviembre de $2001(0.52 \mathrm{~kg} / \mathrm{hora} /$ pescador, $\mathrm{DE}=0.28$ promedio mensual).

El ANOVA (Cuadro 2) no reveló diferencias estadísticamente significativas de CPUE entre los AAs para la época de invierno. Entre las épocas climáticas para Tolú el resultado fue el mismo. Igual ocurrió al comparar la CPUE (promedio mensual) de este estudio con la CPUE encontrada en otros estudios (Cuadro 3 ). Las diferencias no fueron significativas tanto para los hábitats naturales y caladeros tradicionales de pesca, como para los otros hábitats artificiales evaluados en el golfo (Cuadro 2).

Las especies con mayor CPUE, abundancia, peso y frecuencia en los dos AAs fueron Sphyraena guachancho y Scomberomorus brasiliensis. Caranx crysos presentó alta abundancia, peso y frecuencia en Punta de Piedra y Tolú, mientras que Lutjanus synagris solo tuvo alta frecuencia en los dos AAs. Asimismo, Scomberomorus cavalla registró alto peso, y Sarda sarda tuvo alta CPUE y peso en Punta de Piedra y Tolú. Ctenosciaena gracilicirrhus fue abundante en los dos AAs, mientras que Opisthonema oglinum solo lo fue en Tolú (Cuadro 1).

Por otra parte, las principales especies capturadas en los AAs presentaron promedios similares de LE durante el estudio y en cada AA (Fig. 3), con un amplio espectro de tamaños. Según la prueba de Kruskal-Wallis 
CUADRO 1

Composición de la captura, CPUE Total (kg/hora/pescador), peso total en $\mathrm{kg}$, abundancia total (N), y porcentaje de frecuencia $(\%$ F) en el AA de Punta de Piedra y Tolú del Golfo de Morrosquillo

TABLE 1

Catch composition, total CPUE (kg/hour/fisherman), total weight in $\mathrm{kg}$, total abundance (N), and frequency percentage $(\% F)$ in the AR of Punta de Piedra and Tolú from Gulf of Morrosquillo

\begin{tabular}{|c|c|c|c|c|c|c|c|c|}
\hline \multirow{2}{*}{ Especies } & \multicolumn{4}{|c|}{ Punta de Piedra } & \multicolumn{4}{|c|}{ Tolú } \\
\hline & CPUE & Peso & $\mathrm{N}$ & $\% \mathrm{~F}$ & CPUE & Peso & $\mathrm{N}$ & $\% \mathrm{~F}$ \\
\hline Arius sp. & - & - & - & - & 0.00 & 1.2 & 2 & 2 \\
\hline Abudefduf taurus & - & - & - & - & 0.02 & 0.1 & 1 & 1 \\
\hline Bairdiella ronchus & 0.24 & 1.9 & 22 & 16.5 & 0.10 & 3.5 & 29 & 8.5 \\
\hline Balistes capriscus & - & - & - & - & 0.03 & 0.4 & 1 & 1 \\
\hline Caranx crysos & 0.47 & 6.4 & 50 & 41.5 & 5.33 & 167 & 1099 & 80.9 \\
\hline Caranx hippos & - & - & - & - & 0.13 & 5 & 12 & 5.3 \\
\hline Caranx latus & - & - & - & - & 0.01 & 0.3 & 2 & 2 \\
\hline Cathorops mapale & 0.12 & 1.8 & 6 & 4.2 & 0.27 & 11.1 & 36 & 20.2 \\
\hline Centropomus pectinatus & - & - & - & - & 0.01 & 0.6 & 5 & 4.2 \\
\hline Chaetodipterus faber & 0.01 & 0.5 & 3 & 8.3 & - & - & - & - \\
\hline Chloroscombrus chrysurus & 0.04 & 0.7 & 9 & 20.8 & 0.30 & 10.9 & 193 & 40.4 \\
\hline Coryphaena hippurus & 0.05 & 1 & 1 & 4 & 0.68 & 12.5 & 19 & 9.5 \\
\hline Ctenosciaena gracilicirrhus & 0.69 & 7 & 140 & 33.3 & 1.28 & 25.2 & 549 & 47.9 \\
\hline Cynoscion microlepidotus & - & - & - & - & 0.03 & 2 & 4 & 4.2 \\
\hline Dasyatis guttata & - & - & - & - & 0.22 & 7 & 2 & 2 \\
\hline Elagatis bipinnulata & - & - & - & - & 0.05 & 2.3 & 37 & 1 \\
\hline Elops saurus & - & - & - & - & 0.01 & 1.2 & 2 & 2 \\
\hline Epinephelus itajara & - & - & - & - & 4.37 & 70 & 1 & 1 \\
\hline Eucinostomus melanopterus & 0.02 & 0.4 & 9 & 12.5 & 0.05 & 1.7 & 23 & 8.5 \\
\hline Hemiramphus brasiliensis & - & - & - & - & 0.05 & 1.5 & 20 & 3.2 \\
\hline Kyphosus incisor & - & - & - & - & 0.00 & 0.1 & 1 & 1 \\
\hline Lutjanus analis & - & - & - & - & 0.00 & 0.1 & 1 & 1 \\
\hline Lutjanus cyanopterus & - & - & - & - & 0.00 & 0.4 & 1 & 1 \\
\hline Lutjanus synagris & 0.65 & 6.1 & 58 & 50 & 0.73 & 24.9 & 239 & 54.2 \\
\hline Menticirrhus americanus & 0.04 & 0.2 & 1 & 4 & - & - & - & - \\
\hline Mycteroperca venenosa & - & - & - & - & 0.23 & 11 & 2 & 2 \\
\hline Oligoplites saurus & - & - & - & - & 0.18 & 6.5 & 109 & 25.5 \\
\hline Opisthonema oglinum & 0.00 & 0.1 & 6 & 4 & 0.63 & 24.3 & 659 & 42.2 \\
\hline Polydactylus virginicus & 0.04 & 0.6 & 3 & 12.5 & 0.01 & 0.5 & 3 & 2 \\
\hline Pomadasys corvinaeformis & 0.00 & 0.1 & 1 & 4 & 0.09 & 2.5 & 50 & 15.9 \\
\hline Rachycentron canadum & - & - & - & - & 0.01 & 0.7 & 1 & 2 \\
\hline Sarda sarda & 0.57 & 7.6 & 16 & 20.8 & 3.03 & 78.3 & 143 & 35 \\
\hline Scomberomorus brasiliensis & 4.65 & 87.2 & 189 & 79 & 9.40 & 406.1 & 1181 & 59.5 \\
\hline Scomberomorus cavalla & 0.21 & 12.2 & 14 & 12.5 & 7.57 & 303.8 & 410 & 53.2 \\
\hline Selene vomer & - & - & - & - & 0.01 & 0.4 & 11 & 5.3 \\
\hline Sphyraena guachancho & 0.83 & 12 & 80 & 62.5 & 8.23 & 250.2 & 2546 & 82 \\
\hline Sphyrna sp. & 0.02 & 0.5 & 1 & 4 & - & - & - & - \\
\hline Trichiurus lepturus & - & - & - & - & 0.01 & 0.8 & 3 & 3 \\
\hline Tylosurus crocodilus & 0.01 & 0.2 & 1 & 4 & 0.95 & 33.2 & 120 & 46.8 \\
\hline Total & & 146.5 & 610 & & & 1467.3 & 7517 & \\
\hline
\end{tabular}




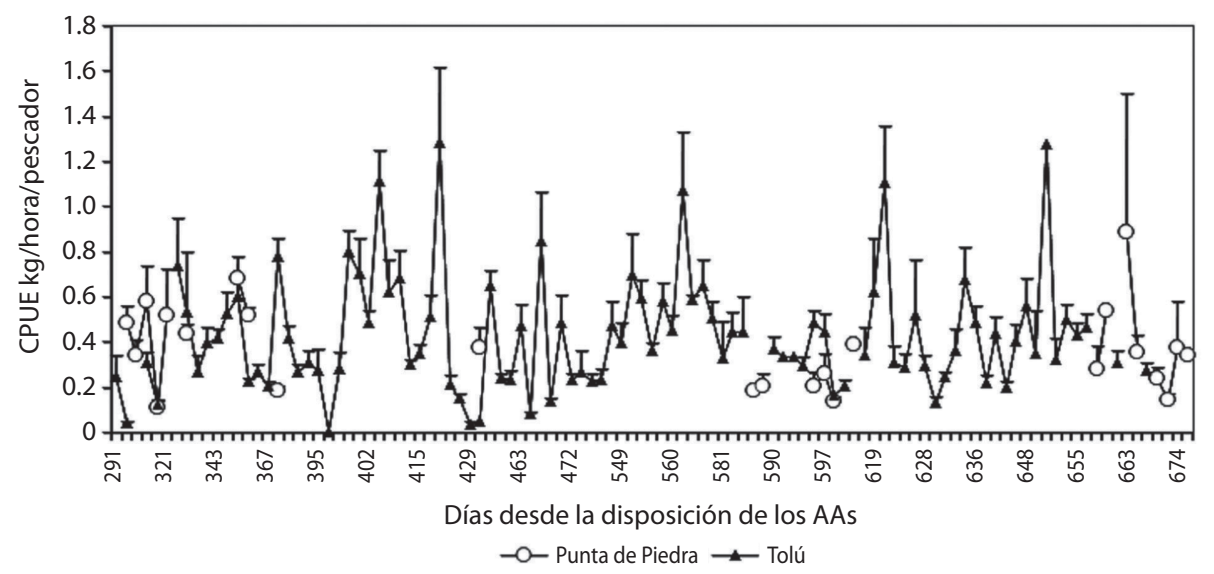

Fig. 2. Valores promedio de CPUE en los AAs durante los días de estudio con la desviación estándar, contados desde la disposición de las estructuras en el Golfo de Morrosquillo.

Fig. 2. CPUE average values in the ARs during the study days with the standard deviation, counted since the deployment of the structures in the Gulf of Morrosquillo.

\section{CUADRO 2}

Análisis de varianza a una vía (ANOVA) para comparar los valores de CPUE entre los AAs para la época de lluvia, entre épocas climáticas para el AA de Tolú, entre los AAs y otros hábitats artificiales y entre los AAs y los hábitats naturales (antes de la instalación de los AAs)

TABLE 2

One-way analysis of variance (ANOVA) to compare the CPUE values: (A) between the ARs in the rainy season, (B) between seasons in the AR of Tolu, (C) between the ARs and other artificial habitats and (D) between the ARs and the natural habitats (before the deploying of the ARs)

\begin{tabular}{|c|c|c|c|c|c|}
\hline Fuente de variación & Suma de Cuadrados & GL & Cuadrado Medio & F-Radio & P-valor \\
\hline \multicolumn{6}{|c|}{ A. Entre los AAs para la época de lluvia } \\
\hline Entre grupos & 0.0039 & 1 & 0.0039 & \multirow{2}{*}{0.01} & \multirow{2}{*}{$0.92 *$} \\
\hline Dentro de los grupos & 47.63 & 115 & 0.41 & & \\
\hline \multicolumn{6}{|c|}{ B. Entre épocas climáticas para el AA de Tolú } \\
\hline Entre grupos & 0.61 & 1 & 0.61 & \multirow{2}{*}{1.37} & \multirow{2}{*}{$0.24 *$} \\
\hline Dentro de los grupos & 40.07 & 90 & 0.44 & & \\
\hline \multicolumn{6}{|c|}{ C. Entre los AA y otros hábitats artificiales } \\
\hline Entre grupos & 0.28 & 1 & 0.28 & \multirow{2}{*}{0.72} & \multirow{2}{*}{0.39} \\
\hline Dentro de los grupos & 17.94 & 46 & 0.39 & & \\
\hline \multicolumn{6}{|c|}{ D. Entre los AAs y los hábitats naturales (antes de la instalación de los AAs) } \\
\hline Entre grupos & 1.12 & 1 & 1.12 & \multirow{2}{*}{1.62} & \multirow{2}{*}{0.21} \\
\hline Dentro de los grupos & 30.63 & 44 & 0.69 & & \\
\hline
\end{tabular}


CUADRO 3

Estimaciones promedio de CPUE (kg/hora/pescador) en hábitats naturales, no-arrecifales (caladeros tradicionales) y hábitats artificiales para pesca con línea de mano durante el día, en canoa de madera y vela en el Golfo de Morrosquillo, recopilados de Ramirez et al. (1994), Herazo \& Torres (1998), SEA (2000), Gámez \& Sáenz-Betancourt (2001) y observaciones personales (*)

TABLE 3

Average estimations of CPUE (kg/hour/fisherman) in natural habitats, non-reef habitats (traditional fishing grounds) and artificial habitats for hook and line fishing during daylight hours, from wood canoe and sail in the gulf of

Morrosquillo, compiled from Ramírez et al. (1994), Herazo \& Torres (1998), SEA (2000), Gámez \& Sáenz-Betancourt (2001) and personal observations (*)

\begin{tabular}{|c|c|c|c|c|c|}
\hline Lugar & CPUE & Hábitat & Lugar & CPUE & Hábitat \\
\hline \multirow[t]{6}{*}{ Porvenir } & 0.35 & No-Arrecifal & Tolú & 0.22 & AA Llantas \\
\hline & 0.24 & No-Arrecifal & & 0.20 & AA Llantas \\
\hline & 0.35 & No-Arrecifal & & 0.25 & AA Llantas \\
\hline & 0.20 & No-Arrecifal & & 0.09 & AA Llantas \\
\hline & 0.13 & No-Arrecifal & & 0.21 & AA Llantas \\
\hline & & & & 0.07 & AA Llantas \\
\hline \multirow[t]{8}{*}{ Tolú } & 0.55 & Arrecife Natural & & 0.10 & AA Llantas \\
\hline & 0.24 & Arrecife Natural & & 0.13 & AA Llantas \\
\hline & 0.43 & No-Arrecifal & & 0.16 & AA Llantas \\
\hline & 0.53 & No-Arrecifal & & 0.33 & AA Llantas \\
\hline & 0.14 & Arrecife Natural & & 0.43 & AA Llantas* \\
\hline & 0.37 & No-Arrecifal* & & 0.21 & AA Piedras* \\
\hline & 0.33 & No-Arrecifal* & & 0.52 & Otro HA* \\
\hline & 0.12 & No-Arrecifal* & & 0.31 & Otro HA* \\
\hline \multirow[t]{6}{*}{ La Caimanera } & 0.31 & No-Arrecifal & La Caimanera & 1.12 & AA Llantas \\
\hline & 0.15 & No-Arrecifal & & 1.10 & AA Llantas \\
\hline & 0.80 & No-Arrecifal & & 0.62 & AA Llantas \\
\hline & 0.60 & No-Arrecifal & & 0.40 & AA Llantas \\
\hline & 0.20 & No-Arrecifal & & 0.51 & AA Llantas \\
\hline & & & & 0.30 & AA Llantas \\
\hline \multirow[t]{6}{*}{ Punta Bolívar } & 0.38 & No-Arrecifal & & 0.38 & AA Llantas \\
\hline & 0.34 & No-Arrecifal & & 0.36 & AA Llantas \\
\hline & 0.17 & No-Arrecifal & & 0.34 & AA Llantas \\
\hline & 0.18 & No-Arrecifal & & 0.75 & AA Llantas \\
\hline & 0.60 & No-Arrecifal & & & \\
\hline & & & Punta Bolívar & 0.24 & DAPs \\
\hline \multirow[t]{2}{*}{ El Boquerón } & 0.60 & Arrecife Natural* & & 0.28 & DAPs \\
\hline & & & & 0.84 & DAPs \\
\hline \multirow[t]{3}{*}{ Bahía de Cispatá } & 0.52 & No-Arrecifal & & 0.99 & DAPs \\
\hline & 0.49 & No-Arrecifal & & 1.02 & DAPs \\
\hline & & & & 0.24 & DAPs \\
\hline \multirow[t]{2}{*}{ Rincón } & 0.70 & Arrecife Natural & & & \\
\hline & 0.19 & Arrecife Natural & & & \\
\hline Berrugas & 0.003 & No-Arrecifal & & & \\
\hline $\mathrm{n}$ & 29 & & $\mathrm{n}$ & 30 & \\
\hline Promedio & 0.35 & & Promedio & 0.42 & \\
\hline $\mathrm{DE}$ & \pm 0.19 & & DE & \pm 0.31 & \\
\hline
\end{tabular}




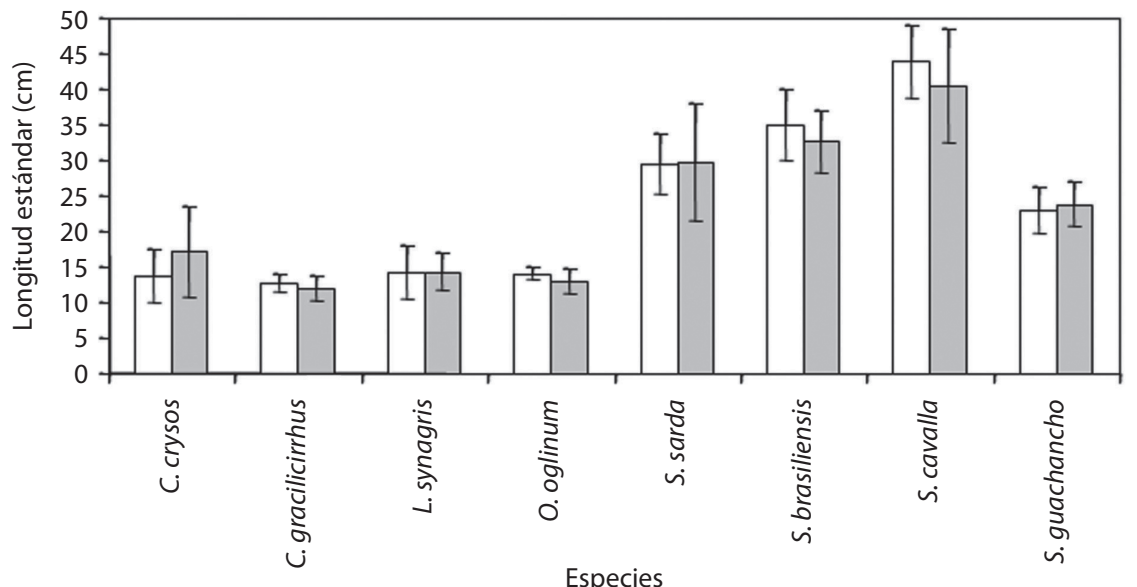

$\square$ Punta de Piedta $\square$ Tolú

Fig. 3. Promedio de longitud estándar con la desviación estándar de las principales especies capturadas en el AA de Punta de Piedra y Tolú, Golfo de Morrosquillo.

Fig. 3. Standard length average with the standard deviation from the main caught species in the AR of Punta de Piedra and Tolú, Gulf of Morrosquillo.

\section{CUADRO 4}

Prueba de Kruskal-Wallis para comparar la longitud estándar de las principales especies capturadas en la época lluviosa de 2001 entre el AA de Punta de Piedra y Tolú con el promedio y rango de tallas en cm en cada AA,

Golfo de Morrosquillo

TABLE 4

Kruskal-Wallis test to compare the standard length of main caught species in the rainy season of 2001 between the AR of Punta de Piedra and Tolú, with average and size range in $\mathrm{cm}$ in each AR, gulf of Morrosquillo

\begin{tabular}{|c|c|c|c|c|c|c|}
\hline \multirow{2}{*}{ Especie } & \multirow{2}{*}{$\mathrm{n}$} & \multirow{2}{*}{ P-valor } & \multicolumn{2}{|c|}{ Punta de Piedra } & \multicolumn{2}{|c|}{ Tolú } \\
\hline & & & Pro. (DE) & Ámbito & Pro. (DE) & Ámbito \\
\hline Caranx crysos & 632 & n.s. & $13.3( \pm 2.5)$ & $9-17$ & $15.6( \pm 4.4)$ & $7-29$ \\
\hline Ctenosciaena gracilicirrhus & 229 & $\mathrm{p}<0.001$ & $12.5( \pm 1.1)$ & $10-15$ & $11.6( \pm 1.7)$ & $8-16$ \\
\hline Lutjanus synagris & 86 & $\mathrm{p}<0.05$ & $13.9( \pm 3.7)$ & $9-26$ & $14.5( \pm 2.5)$ & $9-24$ \\
\hline Scomberomorus brasiliensis & 258 & $\mathrm{p}<0.001$ & $37.4( \pm 3.8)$ & $27-48$ & $34( \pm 5.3)$ & $21-48$ \\
\hline Sphyraena guachancho & 368 & n.s. & $23.1( \pm 2.7)$ & $17-28$ & $23.4( \pm 2.8)$ & $12-32$ \\
\hline
\end{tabular}

(n: tamaño de la muestra; Pro.: promedio; DE: desviación estándar; n.s.: no significativo).

(n: sample size; Pro.: average; DE: standard deviation; n.s.: non significant).

(Cuadro 4), no se encontraron diferencias significativas de talla entre los AAs (época lluviosa) para $C$. crysos y $S$. guachancho, mientras que las especies $C$. gracilicirrhus, L. synagris y $S$. brasiliensis si presentaron diferencias significativas. Entre épocas climáticas en el AA de Tolú, C. crysos, O. oglinum, S. sarda y $S$. guachancho tuvieron diferencias significativas en sus tallas (Cuadro 5).

El análisis de clasificación no reveló segregaciones temporales o espaciales interpretables de los ensamblajes sometidos a la pesca en concordancia con el análisis de varianza que comparó la CPUE (Fig. 4). El promedio de la 
CUADRO 5

Prueba de Kruskal-Wallis para la longitud estándar de las principales especies capturadas en el AA de Tolú entre las dos épocas climáticas, con el promedio y ámbito de tallas en cm, en el Golfo de Morrosquillo

TABLE 5

Kruskal-Wallis test to the standard length of main caught species in the AR of Tolu between the two climatic seasons, with average and size range in $\mathrm{cm}$, at Gulf of Morrosquillo

\begin{tabular}{|c|c|c|c|c|c|c|}
\hline \multirow{2}{*}{ Especies } & \multirow{2}{*}{$\mathrm{n}$} & \multirow{2}{*}{ P-valor } & \multicolumn{2}{|c|}{ Época lluviosa } & \multicolumn{2}{|c|}{ Época seca } \\
\hline & & & Pro. (DE) & Ámbito & Pro. (DE) & Ámbito \\
\hline Caranx crysos & 812 & $\mathrm{p}<0.01$ & $15.6( \pm 2.5)$ & $7-29$ & $19.0( \pm 6.8)$ & $10-32$ \\
\hline Ctenosciaena gracilicirrhus & 549 & n.s. & $11.6( \pm 1.7)$ & $8-16$ & $11.8( \pm 1.5)$ & $9-20$ \\
\hline Lutjanus synagris & 238 & n.s. & $14.5( \pm 2.5)$ & $9-24$ & $14.3( \pm 2.5)$ & $10-24$ \\
\hline Opisthonema oglinum & 659 & $\mathrm{p}<0.001$ & $13.5( \pm 1.5)$ & $10-16$ & $12.8( \pm 1.9)$ & $7-17$ \\
\hline Sarda sarda & 143 & n.s. & $27.9( \pm 6.0)$ & $16-50$ & $31.6( \pm 9.8)$ & $20-55$ \\
\hline Scomberomorus brasiliensis & 1182 & $\mathrm{p}<0.001$ & $34( \pm 5.3)$ & $21-48$ & $32.2( \pm 3.8)$ & $20-60$ \\
\hline Scomberomorus cavalla & 410 & n.s. & $39.8( \pm 7.4)$ & $20-60$ & $41.2( \pm 8.6)$ & $21-77$ \\
\hline Sphyraena guachancho & 2546 & $\mathrm{p}<0.001$ & $23.4( \pm 2.8)$ & $12-32$ & $23.7( \pm 3.2)$ & $13-35$ \\
\hline
\end{tabular}

(n: tamaño de la muestra; Pro.: promedio; DE: desviación estándar; n.s.: no significativo).

(n: sample size; Pro.: average; DE: standard deviation; n.s.: non significant).

temperatura fue $29.5^{\circ} \mathrm{C}$ en los AAs con ámbito entre $27.5^{\circ} \mathrm{C}$ y $32^{\circ} \mathrm{C}(\mathrm{DE}=1.09$ Punta de Piedra; $\mathrm{DE}=1.04$ Tolú), mientras que la salinidad tuvo un valor promedio de 30 en los AAs y osciló entre 25 y 35 (DE=2.69 Punta de Piedra; $\mathrm{DE}=2.78$ Tolú). Ninguna correlación significativa fue encontrada entre los factores bióticos y abióticos (Cuadro 6).

\section{DISCUSIÓN}

Las diferencias en el total de especies capturadas y la biomasa absoluta extraída de los AAs fueron causadas por el mayor esfuerzo ejercido en Tolú, en razón a que Punta de Piedra está a mayor distancia de la costa, lo cual tuvo como resultado que los pescadores prefirieran pescar en lugares diferentes a este AA (obs. pers.). Sin embargo, como se observó en las asociaciones de peces evaluadas por medio de censos visuales (Delgadillo et al. 2004), el AA de Punta de Piedra tuvo mayor abundancia como resultado de un volumen, área y complejidad estructural superior con respecto a Tolú, y esto probablemente sucedería con la pesca si los niveles de esfuerzo en los dos arrecifes fueran similares. Por otra parte, es de anotar que la presencia de algunos bajos naturales y otras estructuras artificiales como llantas y piedras en las cercanías de Tolú y también en Punta de Piedra, pueden conformar un grupo o complejo de hábitats, como propone Bortone et al. (2000). Esto, sumado a las características físicas de los AAs citadas antes, tiene efectos directos positivos en la diversidad, distribución, biomasa y persistencia de las poblaciones (Charbonnel et al. 2002, Sherman et al. 2002, Lan \& Hsui 2006), aspecto importante para las aplicaciones pesqueras y efectividad ecológica de los AAs en el área, cuyo propósito es dar sostenibilidad en el tiempo a los recursos pesqueros, mejorar y diversificar las oportunidades de captura para las comunidades de pescadores, pues según Bohnsack \& Sutherland (1985) el área mínima para propuestas pesqueras está entre $400 \mathrm{~m}^{3}$ y $3000 \mathrm{~m}^{3}$.

La CPUE tuvo niveles similares de variación entre los AAs para una misma época climática y entre épocas climáticas para un mismo AA, como consecuencia probable de estar sometidos a las mismas condiciones generales del clima, el diseño similar e igual disposición de las estructuras. En particular, las diferencias en volumen de los AAs no tuvieron mayor 


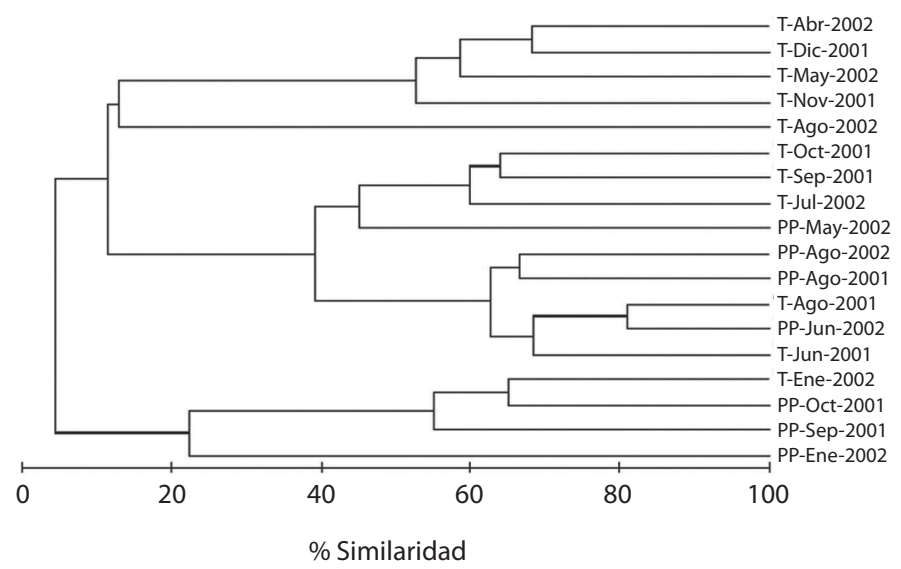

Fig. 4. Dendrograma de similaridad de Bray-Curtis basado en los valores de CPUE mensual en los AAs de Punta de Piedra (PP) y Tolú (T), en el Golfo de Morrosquillo.

Fig. 4. Bray-Curtis similarity dendrogram based in the month CPUE values in the ARs of Punta de Piedra (PP) and Tolú (T), in the Gulf of Morrosquillo.

CUADRO 6

Valor de la correlación por rangos de Spearman entre las variables bióticas y los factores abióticos en los AAs del Golfo de Morrosquillo

TABLE 6

Spearman range correlation value between biotic variables and the abiotic factors in the AR from Gulf of Morrosquillo

\begin{tabular}{|c|c|c|c|c|}
\hline \multirow{2}{*}{ Variable/Factor } & \multicolumn{2}{|c|}{ Punta de Piedra } & \multicolumn{2}{|c|}{ Tolú } \\
\hline & Salinidad & Temperatura & Salinidad & Temperatura \\
\hline CPUE & $-0.17 *$ & $0.25 *$ & $0.02 *$ & $0.07 *$ \\
\hline Especies capturadas & $-0.26 *$ & $0.04 *$ & $-0.17 *$ & $0.05 *$ \\
\hline
\end{tabular}

(*: no significativo). (*: non-significant).

impacto en la CPUE, pues como afirman Kim et al. (2005), se necesita una diferencia del doble para causar una variación significativa de estos valores en los AAs. La similitud de CPUE aquí observada, concuerda con el patrón homogéneo de CPUE en espacio y tiempo registrado en estudios previos de dinámica pesquera en AAs de llantas en el sector (Herazo \& Torres 1998), y para los mismos AAs del presente estudio en términos de los ensamblajes de peces (Delgadillo et al. 2004). Esto tiene relación con las características ambientales estables del golfo a lo largo del año (CIOH 2001), y probablemente con la preferencia de los pescadores por las especies pelágicas que suelen ser muy móviles. Como soporte de esta similitud, el análisis de clasificación no evidenció patrones de asociación por AAs o época climática, y además se observó una falta de relación entre las variables biológicas (CPUE y las especies 
capturadas) con los factores ambientales. De esta forma, es posible que los cambios en algunas características físico-químicas de la columna de agua, en especial por los aportes fluviales en la época de invierno, no tengan ningún efecto en la dinámica de las asociaciones sometidas a la pesca y los valores de CPUE.

Este patrón de similitud sugiere que probablemente dichas asociaciones en los AAs se están acercando a su capacidad de carga, entendida esta como el desarrollo de una actividad pesquera sostenible, en donde la CPUE, abundancia y número de especies extraídas oscilen alrededor de un valor medio relativamente estable. Según Bohnsack \& Sutherland (1985), Pratt (1994) y del Monte-Luna et al. (2004), usualmente esa capacidad se alcanza entre uno y cinco años después de la colocación de los hábitats, dependiendo de las características ambientales, físicas, biológicas y de explotación de los AAs. Sin embargo, una verdadera valoración de esta capacidad debe ser el producto de un experimento con diferentes niveles de mortalidad por pesca.

La CPUE de Tolú y Punta de Piedra no fue diferente de la encontrada en otras estructuras artificiales del sector, si bien la captura absoluta en esas estructuras puede ser mayor en razón a su mayor cercanía a la costa lo que permite visitas mas frecuentes. Por su parte, en los AAs se capturó un número similar de especies en relación con ocho DAPs, pero aproximadamente el doble respecto a AAs de llantas, arrecifes naturales y hábitats no arrecifales (Herazo \& Torres 1998, SEA 2000, Gámez \& Sáenz-Betancourt 2001). Esto es resultado de la mayor complejidad estructural (perfil vertical alto y refugios demersales) de los arrecifes, junto a la presencia de otros HAs en sus cercanías que proporcionaron un ambiente heterogéneo e incrementaron su potencial para atraer y mantener diferentes especies, además de los efectos de la cadena y la boya, que actuaron como DAPs (Delgadillo et al. 2004), lo cual ha sido observado en otras estructuras por diversos autores (Beets 1989, Kellison \& Sedberry 1998, Rilov \& Benayahu 2002, Santos \& Monteiro 2007), y soportan la idea del incremento en la complejidad estructural como aspecto preponderante para el diseño de los AAs en busca de favorecer las necesidades ecológicas de las especies objetivo.

Por otro lado, en comparación con los arrecifes naturales, hábitats no arrecifales y caladeros tradicionales de pesca, los valores de CPUE registrados en esta investigación $(0.41 \mathrm{~kg} /$ hora $/$ pescador, promedio para ambos AAs), se encontraron por encima del promedio de CPUE $(0.35 \mathrm{~kg} /$ hora/pescador) de reportes previos (Ramírez et al. 1994, SEA 2000), lo cual refuerza la idea de los AAs como concentradores de biomasa, en probable combinación con el agotamiento de los sitios tradicionales de pesca. Es de notarse que la biomasa extraída de los AAs en comparación con los hábitats arrecifales y no arrecifales naturales, debe ser más alta si se considera la pesca nocturna e ilegal (arrastres y trasmallos), no estimada en este estudio. No obstante, los AAs fueron claramente efectivos en mejorar (respecto a hábitats naturales) y mantener (respecto a otros hábitats artificiales) la CPUE y el número de especies, así como en la creación de una localidad alternativa de pesca como ya se ha documentado en otras partes en el mundo (McGlennon \& Branden 1994, Pickering \& Whitmarsh 1997, Dempster \& Taquet 2004, Santos \& Monteiro 2007). Es importante aclarar que esta mejora y mantenimiento es generada principalmente por la agregación de biomasa a los AAs y no por la producción in situ, aspectos que se discutirán con detalle más adelante.

Las principales especies capturadas en los AAs fueron igualmente registradas sobre otras estructuras y ambientes naturales del área (Ramírez et al. 1994, Herazo \& Torres 1998, Sandoval 2000, SEA 2000, Gámez \& SáenzBetancourt 2001, Herazo et al. 2006). La LE promedio de C. crysos, S. sarda, S. brasiliensis y $S$. guachancho fue menor a la registrada en estos estudios, mientras que C. gracilicirrhus, L. synagris, O. oglinum y $S$. cavalla presentaron valores similares. Este patrón pone en evidencia la constante presión a la que están y han estado expuestas históricamente las especies comerciales y de la pesca acompañante. Sumado 
a esto, $C$. crysos, $S$. sarda, $S$. brasiliensis, $S$. guachancho, L. synagris, y $S$. cavalla probablemente están siendo capturadas por debajo de la talla mínima de madurez sexual (Cervigón 1991, Franco 1992, Gómez et al. 2001, Froese $\&$ Pauli 2008), aspecto que requiere un estudio detallado de las poblaciones locales, que hasta donde es de nuestro conocimiento no se han hecho. Estos resultados aluden una vez más un nivel de amenaza importante a la capacidad de mantenimiento de los stocks y la productividad del área.

Por otro lado, la comparación de LE entre épocas climáticas y AAs para las especies comerciales no reveló un patrón general u homogéneo, lo cual sugiere que la historia de vida de éstas se ve afectada por la presencia de los AAs y la pesca sobre una base individual. Presumiblemente, estos AAs de alto perfil vertical con conexión a la superficie por la boya pueden funcionar como zonas de protección y alimentación para aquellas especies que se capturan en las menores tallas (L. synagris, $O$. oglinum, C. crysos y C. gracilicirrhus), mientras que para las especies de mayor tamaño con hábitos migratorios en general ( $S$. brasiliensis, S. cavalla, S. sarda, S. guachancho), los hábitats actúan como lugares de descanso, alimentación (presencia de carnada), referencia geográfica o recomposición de cardúmenes, como afirman Castro et al. (2002). En concordancia con la anterior idea, el incremento en la composición de especies pelágicas y demersales, debido a la atracción de peces presa como anchoas y clupeidos, por el alto perfil de las estructuras en comparación con los otros hábitats y ambientes, es un soporte de la utilidad de los AAs en la expansión de las oportunidades pesqueras, además de señalar su potencial como herramienta de manejo pesquero. Asimismo, el esfuerzo pesquero sobre los ambientes naturales probablemente fue reducido al tiempo que este se dirigía hacia los AAs, lo cual no fue cuantificado y requiere estudios posteriores para establecer si el esfuerzo pesquero es desplazado a los nuevos AAs.

Un aspecto negativo potencial de los AAs que debe ser considerado en su utilización, es el incremento a la vulnerabilidad por pesca de especies con lento crecimiento y reproducción como meros y chernas (Jennings \& Lock 1996), así como estadios sensibles de las especies pelágicas que son atraídas a las estructuras, especialmente cuando la intensidad pesquera es alta. Los datos presentados en esta investigación son insuficientes para resolver el controversial problema de atracción vs. producción, aunque esto se vuelve un tópico irrelevante cuando las estructuras son instaladas en zonas con ambientes arrecifales naturales escasos, alejados o inexistentes, en donde la adición de sustratos artificiales resulta en una transformación de la biomasa de poco interés para la pesca a una biomasa valorada (Shipp \& Minton 2005), como probablemente ocurre en el golfo. No obstante, el patrón observado de mejorar (respecto a hábitats naturales) y mantener (respecto a otros HAs y AAs) es ocasionado por la agregación de los peces y no por la producción de biomasa en las estructuras.

Si bien los AAs generan un nuevo hábitat en el cual las especies pueden refugiarse, alimentarse y tener un crecimiento somático y gonadal como fue experimentalmente observado por DeMartini et al. (1994), así como promover el asentamiento, desarrollo y supervivencia de organismos juveniles, además de la evidencia circunstancial de reproducción (Delgadillo et al. 2004), la presión pesquera ejercida sobre las estructuras puede acelerar la sobreexplotación y reducción de los stocks, si estas no producen biomasa efectivamente (Polovina 1989, Grossman et al. 1997). Lo anterior sugiere la elaboración de estudios integrales que aborden la diversidad de procesos que afectan las asociaciones en los AAs (Lindberg 1997), pues algunos autores han encontrado un aumento considerable de la producción general en un área determinada tanto para la biomasa explotable como no explotable y bajo diferentes escenarios de utilización, mientras que otros no han observado una clara evidencia al respecto (Pickering \& Whitmarsh 1997, Bayle-Sempere et al. 2001, Powers et al. 2003, Santos \& Monteiro 2007). 
De cualquier forma, un posible paliativo para evitar los efectos negativos generados por la pesca, es el cerramiento de una proporción de AAs o la creación de áreas marinas protegidas incorporando AAs (Pitcher \& Seaman 2000, Claudet \& Pelletier 2004) con el fin de preservar y resguardar a las diferentes especies asociadas, y proporcionar una adecuada utilización de los AAs.

En conclusión, los AAs tuvieron resultados favorables para la comunidad pesquera artesanal del sector, no sin generar igualmente un impacto evidente sobre el recurso, el cual puede ser minimizado con claros parámetros de manejo y con la realización de investigaciones y monitoreos bien planeados.

\section{AGRADECIMIENTOS}

Esta investigación fue posible gracias al apoyo económico y logístico de la Empresa Colombiana de Petróleos (ECOPETROL), al desaparecido Instituto Nacional de Pesca y Acuacultura (INPA), la Corporación Autónoma Regional de Sucre (CARSUCRE) y la colaboración de la comunidad de pescadores artesanales de la región. Agradecemos a Jackeline Correa Schonewoolf por su colaboración en la recolección de los datos en campo.

\section{RESUMEN}

Entre los meses de agosto de 2001 y agosto de 2002 se hizo el seguimiento de la actividad pesquera en dos arrecifes artificiales (AAs) instalados en el Golfo de Morrosquillo, con el fin de evaluar sus posibles impactos en la dinámica pesquera del área. Se determinó la captura por unidad de esfuerzo (CPUE) y su composición, registrando un total de 19 especies en Punta de Piedra y 36 en Tolú con 16 especies en común. La CPUE fue de $0.40 \mathrm{~kg} / \mathrm{hora} /$ pescador en Punta de Piedra y de $0.42 \mathrm{~kg} /$ hora/pescador en Tolú, y la biomasa extraída total estimada fue de $1830 \mathrm{~kg}$ y 4 820kg en Punta de Piedra y Tolú respectivamente, para pescadores que trabajaron con línea de mano en horas diurnas. No se encontraron diferencias significativas de CPUE entre los AAs y no fue evidente ningún patrón estacional. Las diferencias en composición y biomasa extraída total entre los AAs fueron principalmente consecuencia del mayor esfuerzo ejercido en Tolú, mientras que la ausencia de tendencias y correlaciones de los factores abióticos con la CPUE y las especies capturadas, sugiere que los
AAs están cerca de su capacidad de carga. Como era de esperarse, los AAs mantuvieron y mejoraron la CPUE en comparación con valores reportados en hábitats naturales, caladeros tradicionales de pesca y algunos hábitats artificiales del sector, debido a su mayor volumen, complejidad estructural y perfil vertical. Sin embargo, este incremento puede ser el resultado de la agregación de biomasa más que la producción en los AAs, lo cual tiene impactos negativos potenciales sobre el ambiente que necesitan ser evaluados con el desarrollo de investigaciones experimentales. La disposición de AAs no-extractivos y el uso controlado para propuestas pesqueras, puede ser una herramienta útil para el enriquecimiento y manejo de los recursos en el área.

Palabras clave: arrecifes artificiales (AAs), captura por unidad de esfuerzo (CPUE), dinámica pesquera, pesca diurna, Golfo de Morrosquillo.

\section{REFERENCIAS}

Bayle-Sempere, J.T., A.A. Ramos-Esplá \& J.A. Palazón. 2001. Análisis del efecto producción - atracción sobre la ictiofauna litoral de un arrecife artificial alveolar en la reserva marina de Tabarca (Alicante). Bol. Inst. Esp. Oceanogr. 17: 73-85.

Beets, J. 1989. Experimental evaluation of fish recruitment to combination of fish aggregating devices and benthic artificial reefs. Bull. Mar. Sci. 44: 973-983.

Bohnsack, J. 2005. Artificial reefs: paradise found or lost?, p. 2 In J.H. Cowan Jr. (ed.) 8th 8th Int. Conf. Artificial Reefs and Artificial Habitats (CARAH). Biloxi, Mississippi, EEUU.

Bohnsack, J. \& D. Sutherland. 1985. Artificial reef research; a review with recommendations for future priorities. Bull. Mar. Sci. 37: 11-39.

Bortone, S., M. Samoilys \& P. Francour. 2000. Fish and macroinvertebrate evaluation methods, p. 127-164. In W. Seaman Jr. (ed.). Artificial reef evaluation with application to natural marine habitats. CRC, Boca Ratón, Florida, EEUU.

Botsford, L.W., J.C. Castilla \& C.H. Peterson. 1997. The Management of fisheries and marine ecosystems. Science 277: 509-515.

Castro, E. \& D. Stephens. 2000 Validación y ajuste de la tecnología de los mecanismos agregadores de peces en la Isla de San Andrés (Caribe Colombiano). Tesis de especialización. Universidad INCCA de Colombia, Bogotá, Colombia.

Castro, J.J., J.A. Santiago \& A.T. Santana-Ortega. 2002. A general theory on fish aggregation to floating objects: 
an alternative to the meeting point hypothesis. Rev. Fish Biol. Fish. 11: 255-277.

Cervigón, F. 1991. Los peces marinos de Venezuela. Caracas, Venezuela.

Charbonnel, E., C. Serre, S. Ruitton, J-G. Harmelin \& A. Jensen. 2002. Effects of increased habitat complexity on fish assemblages associated with large artificial reef units (French Mediterranean coast). ICES J. Mar. Sci. 59: 208-213.

CIOH. 2001. Caracterización oceanográfica y metereológica del Golfo de Morrosquillo. Centro de Investigaciones Oceanográficas e Hidrográficas (CIOH). Cartagena, Colombia.

Clark, K.R. \& R.M. Warwick. 2001. Changes in marine communities. An approach to statistical analysis and interpretation. PRIMER-E, Plymouth, Inglaterra.

Claudet, J. \& D. Pelletier. 2004. Marine protected areas and artificial reefs; a review of the interactions between management and scientific studies. Aquat. Living Resour. 17: 129-138.

Delgadillo, O., C.B. García \& J. Correa. 2004. Dinámica temporal de la asociación de peces en dos arrecifes artificiales del Golfo de Morrosquillo, Caribe Colombiano. Actual. Biol. 26: 219-230.

Delgadillo, O. 2005. Motile macroinvertebrates assemblages in two artificial reefs of the Morrosquillo gulf, Colombian Caribbean, p. 109. In J.H. Cowan Jr. (ed.) 8th Int. Conf. Artificial Reefs and Artificial Habitats (CARAH). Biloxi, Mississippi, EEUU.

Del Monte-Luna, P., B. Brook, M. Zetina-Rejón \& V. CruzEscalona. 2004. The carrying capacity of ecosystems. Global Ecol. Biogeogr. 13: 485-495.

DeMartini, E.E., A.M. Barnett, T.D. Johnson \& R.F. Ambrose. 1994. Growth and production estimates for biomass-dominant fishes on a Southern California artificial reef. Bull. Mar. Sci. 55: 484-500.

Dempster, T. \& M. Taquet. 2004. Fish aggregation device (FAD) research: gaps in current knowledge and future directions for ecological studies. Rev. Fish Biol. Fish. 14: 21-42.

Essington, T., A. Beaudreau \& J. Wiedenmann. 2006. Fishing through marine food webs. P. Natl. Acad. Sci. USA, 103: 3171-3175.

FAO. 1997. Technical guidelines for responsible fisheries 4. Roma, Italia.
Franco, L. 1992. Maduración sexual y fecundidad del carite (Scomberomorus maculatus) de las costas del estado Falcón, Venezuela. Zootecnia Tropical 10: 157-169.

Gámez, L. \& H. Sáenz-Betancourt. 2001. Evaluación de la sucesión inicial de la ictiofauna asociada a dispositivos agregadores de peces (DAP's), en aguas costeras del Golfo de Morrosquillo, Caribe Colombiano. Tesis de Licenciatura. Universidad de Bogotá Jorge Tadeo Lozano, Santa Marta, Colombia.

García, A. \& B. Navarro. 1998. Diseño, construcción y evaluación de un dispositivo agregador de peces pelágicos (DAP). Tesis de Licenciatura. Universidad del Magdalena, Santa Marta, Colombia.

Gómez, G., R.Guzmán \& R. Chacón. 2001. Parámetros reproductivos y poblacionales de Lutjanus synagris en el Golfo de Paria, Venezuela. Zootecnia Tropical 19: 335-357.

Grossman, G.D., G.P. Jones \& W.J. Seaman Jr. 1997. Do artificial reefs increase regional fish production? A review of existing data. Fisheries 22: 17-23.

Herazo, D. \& A. Torres. 1998. Evaluación de la efectividad pesquera de dos arrecifes artificiales ubicados en el Golfo de Morrosquillo, Caribe Colombiano. Tesis de Licenciatura. Universidad de Bogotá Jorge Tadeo Lozano, Santa Marta, Colombia.

Herazo, D., A. Torres \& E. Olsen. 2006. Análisis de la composición y abundancia de la ictiofauna presente en la pesca del camarón rosado (Penaeus notialis) en el Golfo de Morrosquillo, Caribe Colombiano. Revista MVZ Córdoba 11: 47-61.

Jennings, S. \& J. Lock. 1996. Population and ecosystem effects of reef fishing, p. 193-218. In N.V.C. Polunin \& C.M. Roberts (eds.). Reef fisheries. Chapman \& Hall, Londres, Inglaterra.

Kellinson, G. \& G. Sedberry. 1998. The effects of artificial reef vertical profile and hole diameter on fishes off South Carolina. Bull. Mar. Sci. 62: 763-780.

Kim, C., H. Kim, H. Kakimoto \& W. Seaman Jr. 2005. Optimum size of a reef set in Korea, p. 34 In J.H. Cowan Jr. (ed.) 8th Int. Conf. Artificial Reefs and Artificial Habitats (CARAH). Biloxi, Mississippi, EEUU.

Kochane, I. 2000. El manglar en el Golfo de Morrosquillo, p. 33-43. En L. Niño, M. Rodríguez \& Z. Sáenz (eds.) Ambiente y desarrollo en el Caribe colombiano. PUJUJTL (IDEADE). Cartagena de Indias, Colombia.

Lan, C-H. \& C-Y. Hsui. 2006. Insight from complexity: a new approach to designing the development of artificial reef communities. Bull. Mar. Sci. 78: 21-28. 
Lindberg, W.J. 1997. Can science resolve the attractionproduction issue? Fisheries 22: 10-13.

Ludwig, J. \& J. Reynolds. 1988. Statistical ecology. A primer on methods and computing. Wiley, Nueva York, Nueva York, EEUU.

McGlennon, D. \& K. Branden. 1994. Comparison of catch and recreational anglers fishing on artificial reef and natural seabed in Gulf St. Vincent, South Australia. Bull. Mar. Sci. 55: 510-523.

Molina, A., C. Molina, L. Giraldo, C. Parra \& P. Chevillot. 1994. Dinámica marina y sus efectos sobre la geomorfología del Golfo de Morrosquillo. Boletín Científico CIOH 14: 93-112.

Patiño, F. \& F. Flórez. 1993. Estudio ecológico del Golfo de Morrosquillo. Universidad Nacional de Colombia. Fondo FEN, Bogotá, Colombia.

Pauly, D., V. Christensen, J. Dalsgaard, R. Froese \& F. Torres. 1998. Fishing down marine food webs. Science 279: $860-863$.

Pickering, H. \& D. Whitmarsh. 1997. Artificial reefs and fisheries exploitation: a review of the "attraction versus production" debate, the influence of design and its significance for policy. Fish. Res. 31: 39-59.

Pitcher, T.J., \& W. Seaman Jr. 2000. Petrarch's Principle: how protected human-made reefs can help the reconstruction of fisheries and marine ecosystems. Fish Fish.1: 73-81.

Polovina, J.J. 1989. Artificial reef: nothing more than benthic fish aggregators. CalCOFI Rep. 30: 37-39.

Powers, S.P., J.H. Grabowski, C.H. Peterson \& W.J. Lindberg. 2003. Estimating enhancement of fish production by offshore artificial reefs: uncertainty exhibited by divergent scenarios. Mar. Ecol. Progr. 264: 265-277.

Pratt, J.R. 1994. Artificial habitats and ecosystem restoration: managing for the future. Bull. Mar. Sci. 55: 268-275.

Ramírez, A., O. Sánchez, L. Borrero \& L. Sánchez. 1994. Introducción a la biología pesquera del Golfo de Morrosquillo y su relación con los ecosistemas naturales. Empresa Colombiana de Petróleos, ECOPETROL, Distrito Caño Limón-Coveñas. Colombia.

Rilov, G. \& Y. Benayahu. 2002. Rehabilitation of coral reef fish communities: the importance of artificial reef relief to recruitment rates. Bull. Mar. Sci. 70: 185-197.
Sánchez-Páez, H., G.A. Ulloa-Delgado, H.A. Tavera-Escobar \& W.O. Gil-Torres. 2005. Plan de manejo integral de los manglares de la zona de uso sostenible del sector estuarino de la bahía de Cispatá, Departamento de Córdoba-Colombia. Corporación Autónoma Regional de los Valles del Sinú y del San Jorge-CVS. Corporación Nacional de Investigación y Fomento Forestal-CONIF. Montería, Colombia.

Sandoval, J.H. 2000. Contribución al conocimiento de la pesca costera en el Golfo de Morrosquillo-Mar Caribe Colombiano, p. 119-136. En L. Niño, M. Rodríguez \& Z. Sáenz (eds). Ambiente y desarrollo en el Caribe colombiano. PUJ-UJTL (IDEADE), Cartagena de Indias, Colombia.

Santos, M.N. \& C.C. Monteiro. 2007. A fourteen-year overview of the fish assemblages and yield of the two oldest Algarve artificial reefs (southern Portugal). Hydrobiologia. 580: 225-231.

SEA. 2000. Monitoreo de biología pesquera en el Golfo de Morrosquillo. Empresa Colombiana de Petróleos, ECOPETROL. Distrito Caño Limón-Coveñas, Sucre, Colombia.

Seaman, Jr. W. \& A.C. Jensen. 2000. Purposes and practices of artificial reef evaluation, p. 1-9. In W. Seaman Jr. (ed.). Artificial reef evaluation with application to natural marine habitats. CRC, Boca Ratón, Florida, EEUU.

Sherman, R.L., D.S. Gilliam \& R.E. Spieler. 2002. Artificial reef design: void space, complexity, and attractants. ICES J. Mar. Sci. 59: 196-200.

Shipp, R.L. \& R.V. Minton. 2005. The irrelevance of the attraction-production debate, p. 101. In J.H. Cowan Jr. (ed.) 8th Int. Conf. Artificial Reefs and Artificial Habitats (CARAH). Biloxi, Mississippi, EEUU.

Underwood, A.J. 1997. Experiments in ecology: their logical design and interpretation using analysis of variance. Cambridge, Cambridge, Inglaterra.

Zar, J.H. 1996. Biostatistical analysis. Prentice Hall, Upper Saddle River, Nueva Jersey, EEUU.

\section{REFERENCIAS DE INTERNET}

Froese, R. \& D. Pauly. 2008. FishBase. World Wide Web electronic publication. Kiel, Schleswig-Holstein, Germany. (Downloaded: abril 25 de 2008, http:// www.fishbase.org versión 03/2009).

Terrazul. 2006. Conservación Terrazul. Fundación para la diversidad biológica, Bogotá, Colombia. (ConsultadO: noviembre 15 de 2007, http://terrazul.org/ proyectos.htlm). 
